\title{
A New Window on Child Prefrontal Functions: Inhibition of a Non-Strategic Alternation-Pointing Scheme
}

O. Houdé, N. Angard, B. Pillon and B. Dubois

\author{
(2) OpenEdition \\ Journals \\ Electronic version \\ URL: http://journals.openedition.org/cpl/229 \\ DOI: $10.4000 / \mathrm{cpl} .229$ \\ ISSN: $1379-6100$ \\ Publisher \\ Centre PsyCLÉ
}

\section{Printed version}

Date of publication: 1 August 2001

Electronic reference

O. Houdé, N. Angard, B. Pillon and B. Dubois, « A New Window on Child Prefrontal Functions: Inhibition of a Non-Strategic Alternation-Pointing Scheme », Current psychology letters [Online], 2001/2, 5 | 2001, Online since 16 September 2003, connection on 08 September 2020. URL : http://

journals.openedition.org/cpl/229; DOI : https://doi.org/10.4000/cpl.229

This text was automatically generated on 8 September 2020

(c) All rights reserved 


\section{A New Window on Child Prefrontal Functions: Inhibition of a Non- Strategic Alternation-Pointing Scheme}

O. Houdé, N. Angard, B. Pillon and B. Dubois 